Type of the Paper (Review)

\title{
Parenting and the serotonin transporter gene (5HTTLPR), which association? A
}

\section{systematic review of the literature}

\author{
Marta Landoni ${ }^{1,2 *}$, Alice Dalla Muta ${ }^{2}$, Sonia Di Tella ${ }^{2}$, Giulia Ciuffo ${ }^{1}$, Paola Di Blasio ${ }^{1,2}$, Chiara Ionio ${ }^{1,2}$ \\ 1 CRIdee, Università Cattolica del Sacro Cuore, Milan, Italy \\ 2 Department of Psychology, Università Cattolica del Sacro Cuore, Milan, Italy \\ * Correspondence: marta.landoni@unicatt.it
}

\begin{abstract}
The current systematic review examines whether there is an association between the genetic 5-HTTPLR polymorphism and parenting and the mechanisms by which this association operates. The literature was searched in various databases such as PubMed, Scopus and ScienceDirect. In line with our inclusion criteria, nine articles were eligible out of 22. Most of the studies, analysed in this review, found an association between 5HTTLPR and parenting. Four studies found a direct association between 5-HTTLPR and parenting with conflicting findings: two studies found that mothers carrying the short variant were more sensitive to their infants, while two studies found that parents carrying the $S$ allele were less sensitive. In addition, several studies found strong interaction between genetic and environmental factors, such as childhood stress and disruptive child behaviour, quality of early care experiences, poor parenting environment, and quality of environment. Only one study found an association between children's 5HTTLPR and parenting. Parenting can be described as a highly complex construct influenced by multiple factors, including environmental, parenting and child characteristics. According to the studies, maternal 5-HTTLPR polymorphism is most likely associated with sensitive parenting.
\end{abstract}

Keywords: parenting; 5HTTLPR; serotonin transporter; maternal sensitivity; polymorphism; gene $\mathrm{X}$ environment

\section{Introduction}

A large body of research has shown that parenting can have significant effect on child development, both short and long term [1,2]. Specifically, hostile parenting could be considered a risk factor for the development of emotional, cognitive, and physical health problems in children and is also associated with higher rates of insecure attachment [3]. Several studies have specifically examined parenting, which is defined as the capacity to recognize and respond correctly to the signals sent by the kid [4].

Despite the significant role that parental education plays in children's growth, few studies have been conducted to determine its predictors [2]. Indeed, parenting can be described as a highly complex construct that is influenced by numerous factors, such as environmental, educational and child characteristics [5]. In addition to these factors, genetic factors have recently been examined in the literature in relation to parenting. Mothers are often said to have a "maternal instinct" or a "maternal drive," reflecting a widely 
held belief about mothers: there are innate rules, shaped by evolutionary history and embedded in DNA, that drive all mothers to respond to, nurture, and educate their children [6]. Parenting is shaped by various contextual or "external" influences, and much research has been conducted in this area. A small but growing number of studies have addressed the heritable aspects of mothering, looking more closely at genetic variation at the molecular level to determine how DNA might structure parenting. Throughout the literature, several genes have been considered. Specifically, most studies have focused on the relationship between the serotoninergic system and parenting [2].

Although a genetic influence of 5HTTLPR on nurturing behaviour in animals and other mammals is well established in the literature [7], studies in humans are still pending [2]. However, animal studies have shown that 5 -HT plays a role in parental behaviour, suggesting that the serotonin system, either alone or in combination with the dopamine and oxytocin systems, may be a critical modulator of parental behaviour.

Serotonin is a neurotransmitter involved in the regulation and control of various physiological and psychological processes [8]. In this system, the serotonin transporter (SERT or 5-HTT) plays a crucial role in regulating the availability of serotonin in the synaptic space. Therefore, much of the research has focused on the study of the gene responsible for its encoding (SLC6A4) and its polymorphic variants (5-HTTLPR) [9, 10].

The 5-HTTLPR polymorphism is a functional polymorphism that involves the insertion and/or deletion of 44 pairs of nucleotide base pairs, resulting in two genetic variants: the long variant (L) and the short variant (S) [11]. The long variant is associated with higher SERT functionality and consequently lower availability of serotonin in the synaptic space [9]. The S variation, on the other hand, is linked to decrease serotonin transporter gene transcription, resulting in increased serotonin levels in the synaptic cleft [12].

However, studies focusing on the relationship between 5-HTTLPR and parenting have come to different conclusions [2]. While the short (S) variant has been associated with higher maternal sensitivity in some studies $[1,13]$, other researchers have found opposite results $[2,14]$.

To explain this discrepancy, some researchers hypothesised that genetic factors might interact with environmental components through epigenetic mechanisms (gene- $\mathrm{X}$ - environment interactions) $[12,15,16]$.

Considering these aspects, the present review investigated the association between the genetic polymorphism 5-HTTPLR and parenting. Moreover, the study aims to draw attention to the mechanisms moderating/mediating this relationship to better understand the psychological transitions. To identify, evaluate, and synthesize the literature relevant to this topic, two questions two questions guided our search:

1. Has there ever been found a relationship between 5HTTLPR and parental parenting in 
humans?

2. Are there other variables involved in this relationship?

\section{Materials and Methods}

Preferred Reporting Items for Systematic Reviews and Meta-Analysis (PRISMA) guidelines were used to review quantitative research [17]. According to our aims, we based our questions for the literature search strategy, screening phase, and extraction of the final data.

First, screening questions were combined with inclusion and exclusion criteria [18]. Second, for quantitative studies, the acronym PICOS (Population, Intervention, Comparison, Outcome measures, and Study) was used to refine the questions and establish criteria [19].

Inclusion and exclusion criteria were:

Inclusion criteria: Full-text articles that were peer-reviewed; written in English, quantitative and focused on parenting skills.

Exclusion criteria: studies in languages other than English; discussion papers or systematic reviews; studies conducted on animals that do not focus on parents.

PubMed, Scopus, and ScienceDirect were used to find articles with the following search term: 5HTTLPR AND parenting AND polymorphism in title/abstract.

The literature was reviewed to determine whether it was relevant and met the inclusion criteria. Additional articles related to the review topic were found in the reference lists of all included studies.

To determine if a study could be included in this review, the literature was first searched by abstract and title. The search yielded 23 articles. After screening titles and abstracts, 9 articles were included (Figure 1). 


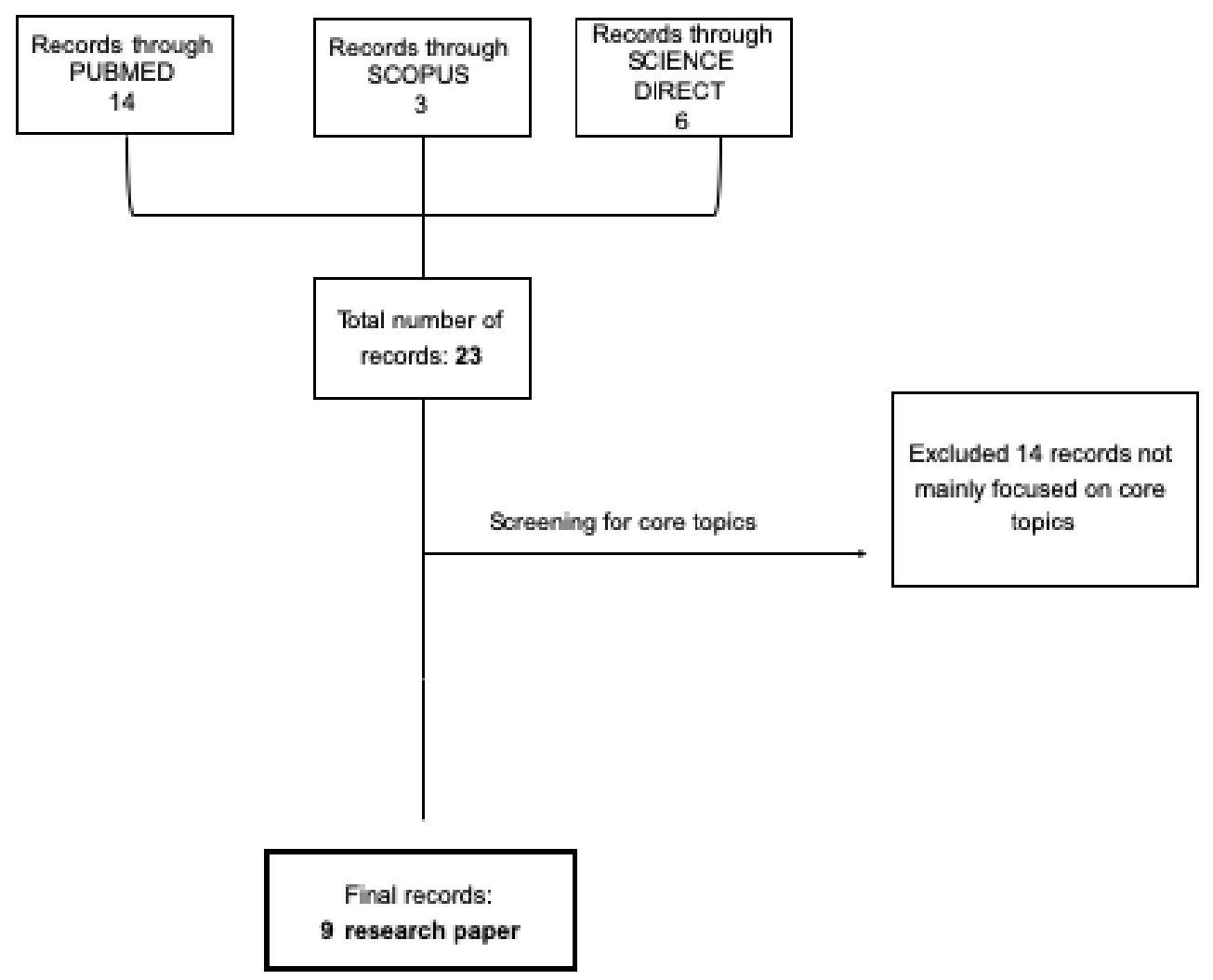

Figure 1. Flow diagram

The assessment of eligibility for inclusion was done in accordance with the PRISMA criteria, and the authors then discussed them to formalize the agreement.

After studies met inclusion criteria, they were assessed using the Genetic Studies Quality Tool (Q-Genie), as presented in Table 1 [20]. This tool is used to assess and detect bias in genetic studies and is divided into nine categories: Research Design, Sample Selection, Exposure Classification, Results Classification, Sources of Bias, Presentation of Statistical Plan, Quality of Statistical Technique, Testing of Assumptions in Genetic Studies, and Interpretation of Results.

To minimise loss of precision and reliability and to account for bias in the results, a Likerttype rating scale with seven categories anchored by 'poor' and 'very good' was used.

Two of the authors performed the scoring (ML and DS) and conferred in case of disagreement.

The main findings of each study were reviewed. The magnitude of the association between parental 5-HTTLPR and maternal sensitivity was assessed using the effect size reported in the article, if available, or calculated from the data reported in the study using 
an online calculator (https://www.psychometrica.de/effect_size.html). Effect sizes were interpreted as follows: for Pearson's $r$ correlation: 0.1 to 0.3 as small effect; 0.3 to 0.5 as medium effect; 0.5 and higher as strong effect; for Cohen's $d, 0.2$ to 0.49 as small effect; 0.5 to 0.79 as medium effect; 0.8 and higher as strong effect; and finally, Cohen (1988) gave guideline values for for $\eta^{2}$ to define small $\left(\eta^{2}=0.01\right)$, medium $\left(\eta^{2}=0.06\right)$, and large $\left(\eta^{2}=0.14\right)$ effects [21].

Table 1. Q-Genie Assessment.

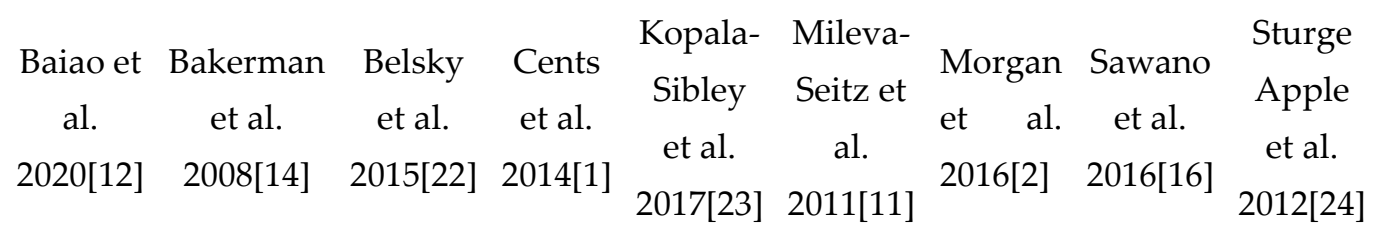

\section{Rationale for study:}

Was a scientific rationale for chosen genes presented 6 6 7

6

6

6

6 5

to avoid selective reporting

of positive results

\section{Selection and definition}

of outcome of interest:

Were the cases appropriately defined?

Were participants appropriately sampled? Were the case/outcome assessors blinded to the

genotype status?

\section{Selection and} comparability of comparison groups

Were the controls appropriately defined?

Were the controls sampled in a way to minimize selection bias?

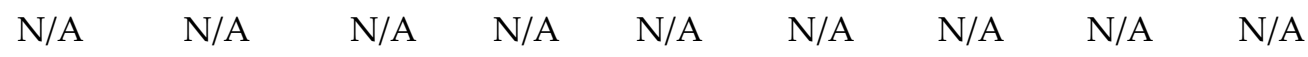
Was a detailed description of selection procedure (i.e. eligibility criteria, sources and methods of ascertainment, methods of matching if 
applicable) outlined or referenced?

\section{Technical classification}

of the exposure

Was the source (e.g. buffy

coat) and method of storage for the DNA sample appropriate?
6

6

5

6

5

6

5

6

Was agreement with the

Hardy Weinberg

equilibrium tested in controls?

\section{Non-technical}

\section{classification of the} exposure

Did a blinded assessor conduct the genotyping?

$$
\text { Was genotyping }
$$

6

5

6

5

6

$5 \quad 6$

conducted in all the

participants from the study

simultaneously or in

smaller

batches?

\section{Other sources of bias}

N/A

5

7

5

6

N/A

N/A

N/A

$\mathrm{N} / \mathrm{A}$

\section{Sample size and power}

Was the sample size

$$
\text { appropriate? }
$$

Was an a priori power analysis conducted?

\section{A priori planning of analyses}

Was the analysis plan

6

$5 \quad 6$

6

6

5

6

appropriate and sufficiently described?

\section{Statistical methods and}

\section{control for confounding}

Were important confounders appropriately controlled?

Were missing data for samples and genetic variant was appropriately 
handled? $>10 \%$ missing genotype data is often unacceptable.

Were the results adjusted for multiple testing to avoid false positive results?

10. Testing of assumptions and

inferences for genetic analyses
all assumptions 6 6 6 $6 \quad 6$ 6 6 6

Were all assumptions concerning the genetic analysis tested?

11. Appropriateness of inferences drawn from 6 6 6 6 6

6

$$
6
$$

5

6 results

Total Score 43

59

62

53

60

50

59

45

54 
Child Behavior Checklist

Teacher Report versions

Social Skills Rating

$$
\text { System }
$$

Cents[1]

\section{7 mother-} child dyads.

Mother-child
interactions were
videotaped

Laboratory

Temperament

Assessment Battery

Preschool Version (Lab-

$\mathrm{TAB})$

Family Assessment

Device (FAD)

the Brief Symptoms

Inven- tory (BSI)

Kopala-

2017
Two independent samples of three-year-olds $(\mathrm{N}=476$ and 405)
Measure of Parenting Styles(MOPS)

Parental Bonding Inventory (PBI)

Maternal support and hostility was recorded
SS/LL/Ls Yes

Repeated measurement analyses revealed that maternal 5-HTTLPR has a consistent main effect on sensitivity; mothers with the S-allele were more sensitive toward their children ( $p=.005)$. The 5 HTTLPR genotype of the child had no bearing on this effect. We found no evidence that the effect of 5HTTLPR on sensitivity was moderated by child social fearfulness.
A child with a short allele on the 5-HTTLPR gene was linked to more maternal hostility and less maternal support, but only when the mother reported poor grandmothers' parenting.

$r=0.17$ \#

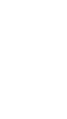


Mileva-Seitz[11] 2011204 mothers Video-recorded motherinfant interaction

Life history calendar (LHC)

Childhood Trauma

Questionnaire (CTQ

Morgan[2]

2016162 parents and their 6- to 9-

year-old

offspring

\section{Dyadic Parent Child Interaction Coding System (DPICS)}

S, LA and Yes The genotype can predict differences in maternal LG sensitivity at 6 months postpartum, even after controlling for maternal age and parity: mothers with a $\mathrm{S}$ (or the functionally similar LG) allele were more sensitive than mothers without the allele during a 30-minute recorded mother-infant interaction. Furthermore, highly significant geneenvironment interactions in relation to maternal behavior were found, such as mothers who lacked the $\mathrm{S}$ or LG alleles orienting away from their babies more frequently if they also reported poor early care quality.

\section{UCLA Life Stress} Interview (LSI)

Parenting Stress IndexShort Form (PSI)

Diagnostic Interview Schedule for Children-

\section{Beck Depression} Inventory-II

SS/LL/Ls Yes The $S$ allele was associated with significantly less observed positive parenting than the LL genotype.

There were also significant gene-environment interactions: Parental negativity was negatively associated with child-related stress in SS/SL genotype parents but not in LL genotype parents; next, observed disruptive child behavior was positively associated with parental negativity in both genotypes, but the effect was strongest in SS/SL parents.
IV

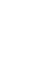

cohen's $d=$

$0.405 \#$ 
Adult ADHD Self-Report

Sawano[16]

2016

93 mothers

PBI

MIBS

Edinburgh Postnatal

Depression Scale (EPDS)

Sturge Apple[24] 2012201 mothers and their two-year old child

Mother-Child Problem

Solving Task videotaped

Mother-Child Free

Play/Compliance Task videotaped

Revised Conflict Tactics Scale (CTS2)

Conflict and ProblemSolving Scale (CPS)

$\begin{array}{cc}\text { s/s } & \text { Yes } \\ \text { l-carriers } & \end{array}$
interaction between the rearing environment and the 5-HTTLPR genotype. In particular, in mothers with homozygous short allele genotype, a poor rearing environment (characterized by low maternal care and high paternal overprotection) reduced positive attitude toward one's own infant. In long allele carriers, on the other hand, this negative effect was almost completely eliminated. Overall, our findings suggest that the 5-HTTLPR gene moderates the impact of maternal parental behavior on the experienced rearing environment, which is consistent with the idea that the short 5-HTTLPR allele amplifies environmental influence.

S/LG/LA Yes genotypes

Mothers with one or two copies of the 5-HTTLPR S allele had a higher risk of both sensitive and harsh/punitive caregiving behaviors. partial $\eta^{2}=$

$0.006^{*}$ 
Iowa Family Interaction

Rating Scales (IFIRS)

Empathetic Awareness

Toward Children's

Needs scales from the

Adult-Adolescent

Parenting Inventory

$$
\text { (AAPI) }
$$

Nurturance scale of the

Parenting Dimensions

Inventory (PDI)

Computerized

Diagnostic Interview

Schedule IV (C DIS IV) 


\section{Results}

As mentioned earlier, the analysis of the nine selected studies revealed a discrepancy in the results (Table 2).

Four of these studies found a direct association between 5-HTTLPR and parenting [1, $2,11,14]$, although the retrieved results varied and the magnitude of the association examined by effect sizes was small. For example, Cents et al.[1] examined whether maternal 5-HTTLPR and sensitivity are associated and if this effect is moderated by the child's 5-HTTLPR genotype or social anxiety.

At 14 months and four years of age, the results revealed a consistent main impact of maternal 5-HTTLPR on maternal sensitivity; women with the $\mathrm{S}$ allele were more sympathetic to their offspring. Moreover, this association was independent of child genotype and social anxiety. These results are consistent with those of Mileva-Seitz et al. [11], who found a direct association between S/LG alleles and more sensitive parenting six months after birth.

In contrast, two studies found a direct association between the $S$ allele and lower parental sensitivity $[2,14]$. The first research[14] indicated that women with the SS genotype were less responsive to their babies than mothers with the LL or LS genotype, even after controlling for differences in maternal education, depression, and marital strife. Morgan et al.[2] found similar outcomes: parents with the $S$ allele (86 percent of moms) had considerably fewer favorable parenting behaviors than parents with the LL-genotype. Moreover, several studies have found strong interactions between genetic and environmental factors [2, 11, 12, 16]. Mileva-Seitz et al.[11] investigated whether genotype and early caregiving experiences were linked to three dimensions of maternal responsiveness (maternal sensitivity, maternal behaviour away from the child, and maternal attitudes-perceived attachment), and discovered highly significant GXE interactions with maternal behaviour and maternal attitudes. Specifically, mothers without $S$ or LG alleles experienced more negative quality of early care and were more likely to distance themselves from their children. Conversely, mothers with S or LG alleles and better quality of early care reported higher scores on ratings of their perceived attachment to their baby. These findings are at odds with the observations of Morgan et al. [2], who found that child-related stress was negatively associated with observed negative parenting in parents with the SS or SL genotype.

Furthermore, in both genotypes, observed disruptive child behavior was positively associated to parental negativity, with the impact being largest in parents with the SS or LS genotype. Sawano et al. [16] reached similar conclusions, finding an interactive effect between parenting environment and 5-HTTLPR on maternal attitudes. Specifically, a 
poor parenting environment (low maternal care and high paternal over-care) was negatively associated with positive attitudes toward their children in mothers with the SS genotype. In contrast, this adverse effect was almost absent in carriers of $\mathrm{L}$ allele.

Different results were obtained by Baio et al.[12], who investigated whether 5-HTTLPR moderates the quality of environmental context on maternal sensitivity. These findings revealed a GXE interaction, with women carrying the SS genotype being more responsive to family environment than $\mathrm{L}$ allele bearers. Depending on whether the environmental setting was of poor or high quality, mothers with the LL or SL genotype showed the greatest and lowest levels of maternal sensitivity. Finally, two studies examined whether children's genotype was related to maternal parenting behaviours and maternal sensitivity [22, 23]. The first research [23] looked at whether the effects of children's 5HTTLPR genotype on their mothers' parenting behaviors were modified by their own parenting experiences. The results supported the possibility of a moderated evocative GXE association, showing that children who carried the $S$ allele experienced higher maternal hostility and lower maternal support, but only when the mother reported lower quality grandmotherly parenting. Belsky et al. [22] investigated whether children's genetic profiles reduce the long- and short-term impacts of early maternal sensitivity on social-emotional and cognitive-linguistic development. Consistent with the observations of Cents et al. [1], the authors found no GXE interaction between children's genotype and maternal sensitivity. Moreover, the children's 5-HTTLPR showed no main effects on social-emotional and cognitive-linguistic development.

\section{Discussion}

The present review aimed to examine the relationship between 5HTTLPR and human parenting, highlighting the role of environment and other variables in this relationship. Furtheraim of this study is to draw attention to the processes that moderate or mediate this link in order to better understand psychological transitions and address the objections raised in the literature.

As noted above, although the literature has demonstrated a genetic influence of 5HTTLPR on parental behaviour in animals and other mammals, very few studies have examined the relationship between $5 \mathrm{HT}$ and parental care in humans [2, 24].

However, the majority of studies included in this review found a relationship between 5HTTLPR and parenting. Four studies showed a direct relationship between 5-HTTLPR and parental caregiving $[1,2,11,14]$, with contrasting results that may prompt future research and hypotheses about different mechanisms acting on this relationship. In addition, several studies found strong interactions between genetic and environmental factors $[2,11,12,16]$, such as infant stress and disruptive infant behaviours[2] , the quality of early care-experiences [11], poor rearing conditions, and the quality of the 
environment [16]. Most psychological traits and behaviors are better explained by a combination of essential characteristics such as gender and socioeconomic status than by genetic markers alone, as Kagan et al.[25] recently argued, though the inclusion of both genetic and environmental factors can predict such outcomes with the greatest accuracy in certain groups. Other authors also investigated whether children's genotype was related to maternal parenting behaviours and sensitivity [1,22,23], but only one study found an association between children's 5HTTLPR and parenting behaviours. Specifically, children carrying the $S$ allele experienced higher maternal hostility and lower maternal support, but only when the mother reported lower quality parenting from the grandmother. These results can be considered consistent with infant twin studies showing that genetically influenced characteristics of children (e.g., temperament) trigger certain parenting behaviours[26].

To explain the nature of the results, there are several explanations. One of them links the 5HTTLPR polymorphism to cognitive aspects. Because the 5-HTTLPR polymorphism is linked to numerous elements of thinking function, it may have an impact on parenting due to its correlations with maternal characteristics. In both rat and human investigations, carriers of the $S$ allele performed better on a variety of tasks, including cognitive flexibility, reversal learning, attention, and inhibition[27,28,29].

To practice empathic parenting, parents must have cognitive flexibility and attention to effectively perceive and respond to their children's signals[4]. For example, studies have shown that mothers with ADHD (attention deficit hyperactivity disorder) tend to show behavioural characteristics of poor parents[30,31]. Reactive parenting styles have also been associated with poor working memory[32]. 5-HTT binding in the putamen and midbrain is influenced by the 5-HTTLPR genotype in humans[33]. As a prominent dopaminergic projection site of the mesocorticolimbic dopamine system, the ventral tegmental area (VTA) in the midbrain is involved in motivation and reward.

Based on these findings, it can be hypothesised that differences in midbrain 5-HTT function are associated with differences in dopamine signalling that influence maternal behaviour. For instance, folate reduces 5 - HT reuptake, affecting maternal behavior in rats [34], and lesions of serotonergic neurotoxins in the median raphe, a key site for serotonin synthesis, reduce suckling and retrieval of pups [34, 35].

Moreover, the 5-HTTLPR polymorphism may have a direct impact on parenting via its influence on maternal traits and neuronal and hormonal consequences. The role of oxytocin and vasopressin in determining parental behavior in various animals appears to be significant $[36,37]$. When serotonin is activated in the hypothalamus, the paraventricular nucleus (PVN) of the hypothalamus releases both chemicals[38]. The PVN also has serotonin receptors. According to research, oxytocin and vasopressin are 
released through serotonin receptors [39]. When oxytocin and vasopressin systems are linked, the 5-HTTLPR may influence maternal sensitivity in parenting.

Parental genetic predisposition can be influenced by the presence or absence of stressful life conditions, with hormonal influences having a greater impact in deprived contexts where social support is lacking[40]. In primates (as opposed to rodents), the scales have tipped in favor of neocortex size and function, according to Numan and Insel[41, 42]. Thus, primate parenting may be driven by cognitive rather than hormonal factors, at least in the absence of extreme conditions [41].

However, the medial preoptic area (MPOA) of the hypothalamus might still be involved in the development of complex voluntary response-strategies by signalling the degree of maternal motivation to the neocortex [41]. For mothers in disadvantaged circumstances, such as those characterised by high levels of stress or marital discord, the links between serotonin and oxytocin system genes and parenting might be even more pronounced. Finally, several authors proposed the differential susceptibility model as an explanation. For example, Hariri et al. [43] found that the $S$ allele was associated with enhanced social cognition and increased amygdala sensitivity to emotional stimuli [44]. Here, it is possible that the $S$ allele serves as a gene of adaptability; it is more beneficial in low-risk circumstances and more challenging in difficult ones $[45,46]$. Because of their greater susceptibility to mood disorders, genotypes containing one or two $\mathrm{S}$ alleles have been termed "vulnerability genotypes." Although it may increase maternal caregiving sensitivity in low-risk contexts by increasing the ability to detect environmental cues, it may also increase the risk of insensitive caregiving and hostility when the environment is more hostile.

Consistent with these observations, the expected direction of the association between 5HTTLPR and parenting is not clear [26]. However, this review has strengthened the understanding of the mechanisms involved in the relationship between genetic components and parenting.

The main limitation of current research on the influence of 5HTTLPR on parenting is the scarcity of studies and the lack of studies based on large samples. Future research could expand our knowledge of this relationship by considering other genotypes that have been identified as potential susceptibility markers (particularly MAOA, BDNF, and MR). Future studies could also consider other factors such as ethnicity as an important moderator in GxE studies, including genetic differential susceptibility studies [26].

\section{Conclusions}

The exploration of the influence of genetics in explaining parenthood only began around the year 2000, so it is a relatively new branch. On the one hand, this is remarkable since 
parenting theory can be considered the first application of evolutionary theory to human development - after Charles Darwin, but before the development of so-called evolutionary psychology.

The genotypes of the parents were identified using molecular genetic techniques. Maternal 5-HTTLPR polymorphism was associated with sensitive parenting. This study adds to the growing body of evidence showing that parenthood is a multifaceted concept. According to Swain et al. [37], parenting is a complex interplay of genes, prior parenting, current experiences, psychological state, neurobiological systems, and environmental conditions. Parenting can be better understood if we acknowledge and provide more insight into the multifactorial processes that underlie it.

Exploring possible mediators of the relationship between 5-HTTLPR and maternal sensitivity, including cognitive flexibility and attention, could provide useful insights into the underlying biological processes and provide further evidence for a link between 5-HTTLPR and parenting

Funding: This research received no external funding

Conflicts of Interest: The authors declare no conflict of interest

\section{References}

1. Cents, R.A., Kok, R., Tiemeier, H., Lucassen, N., Székely, E., Bakermans-Kranenburg, M.J., Hofman, A., Jaddoe, V.W., van IJzendoorn, M.H., Verhulst, F.C., Lambregtse-van den Berg, M.P. Variations in maternal 5-HTTLPR affect observed sensitive parenting. J. Child Psychol. Psychiatry 2014, 55, 1025-32.

2. Morgan, J.E., Hammen, C., Lee, S.S.Parental Serotonin Transporter Polymorphism (5-HTTLPR) Moderates Associations of Stress and Child Behavior with Parenting Behavior. J. Clin. Child Psychol 2016, 47, 76-87.

3. Groh, A.M., Roisman, G.I., van Ijzendoorn, M.H., Bakermans-Kranenburg, M.J., Fearon R.P.The significance of insecure and disorganized attachment for children's internalizing symptoms: a meta-analytic study. Child Dev 2012, 83, 591-610.

4. Ainsworth, M.D.S., Blehar, M.C., Waters, E., Wall, S., Patterns of attachment: A Psychological Study of the Strange Situation. 1978, Erlbaum, Hillsdale, NJ.

5. Belsky, J.The determinants of parenting: A process model. Child Dev 1978, 55, 83-96.

6. Rosenblatt, J.S.Nonhormonal basis of maternal behavior in the rat. Science 1967, 156, 1512-4. 
7. Kuroda, K. O., Tachikawa, K., Yoshida, S., Tsuneoka, Y., \& Numan, M. Neuromolecular basis of parental behavior in laboratory mice and rats: With special emphasis on technical issues of using mouse genetics. Prog. Neuro-Psychopharmacol. Biol. Psychiatry 2011, 35, 1205-1231.

8. Mohammad-Zadeh, L.F., Moses, L., Gwaltney-Brant, S.M. Serotonin: a review. J.Vet.Pharmacol. Ther 2008, 31, 187-199.

9. Kenna, G.A., Roder-Hanna, N., Leggio, L., Zywiak, W.H., Clifford, J., Edwards, S., Kenna, J.A., Shoaff, J., Swift, R.M.Association of the 5-HTT gene-linked promoter region (5-HTTLPR) polymorphism with psychiatric disorders: review of psychopathology and pharmacotherapy. Pharmgenomics Pers. Med. 2012, 5, 19-35.

10. Caspi, A., Hariri, A.R., Holmes, A., Uher, R., Moffitt, T.E. Genetic sensitivity to the environment: the case of the serotonin transporter gene and its implications for studying complex diseases and traits. Am. J. Psychiatry 2010, 167, 509-527.

11. Lesch, K.P., Bengel, D., Heils, A., Sabol, S.Z., Greenberg, B.D., Petri, S., Benjamin, J., Müller, C.R., Hamer, D.H., Murphy, D.L. Association of anxiety-related traits with a polymorphism in the serotonin transporter gene regulatory region. Science 1996, 274, 1527-1531.

12. Baião, R., Fearon, P., Belsky, J., Teixeira, P., Soares, I., Mesquita, A. Does 5-HTTLPR moderate the effect of the quality of the environmental context on maternal sensitivity? Testing the maternal susceptibility hypothesis. Psychiatr. Genet 2020, 30, 49-56.

13. Mileva-Seitz, V.R., Kennedy, J., Atkinson, L., Steiner, M., Levitan, R., Matthews, S.G., Meaney, M.J., Sokolowski, M.B., Fleming, A.S.Serotonin transporter allelic variation in mothers predicts maternal sensitivity, behavior and attitudes toward 6-month-old infants. Genes Brain Behav 2011, 10, 325-33.

14. Bakermans-Kranenburg, M. J., van Ijzendoorn, M. H. Oxytocin receptor (OXTR) and serotonin transporter (5-HTT) genes associated with observed parenting. Soc Cogn Affec Neurosci 2008, 3, 128-134.

15. Mileva-Seitz, V.R., Bakermans-Kranenburg, M.J., van IJzendoorn, M.H. Genetic mechanisms of parenting. Horm. Behav 2008, 77, 211-23.

16. Sawano, E., Doi, H., Nagai, T., Ikeda, S., Shinohara, K.Interactive effects of 5-HTTLPR genotype and rearing environment on affective attitude towards own infant in Japanese mothers. Behav Brain Res $2008,325,173-180$. 
17. Moher, D., Liberati, A., Tetzlaff, J., Altman, D.G.Preferred reporting items for systematic reviews and meta-analyses: the PRISMA statement. PLoS Med 2009, 6.

18. Aromataris, E., Pearson, A.The systematic review: an overview. Am J Nurs 2014, 114, 53-8.

19. Methley, A.M., Campbell, S., Chew-Graham, C., McNally, R., Cheraghi-Sohi, S.PICO, PICOS and SPIDER: a comparison study of specificity and sensitivity in three search tools for qualitative systematic reviews. BMC Health Serv. Res. 2014, 14, 579.

20. Sohani, Z.N., Sarma, S., Alyass, A., et al. Empirical evaluation of the Q-Genie tool: a protocol for assessment of effectiveness. BMJ Open 2016;6,e010403.

21. Cohen, J. Statistical Power Analysis for the Behavioral Sciences. 1988, New York, NY: Routledge Academic.

22. Belsky, J., Newman, D., Widaman, K., Rodkin, P., Pluess, M., Fraley, R., Berry, D., Helm, J.L., Roisman, G.I.Differential susceptibility to effects of maternal sensitivity? A study of candidate plasticity genes. Dev. Psychopathol 2015, 27, 725-746.

23. Kopala-Sibley, D.C., Hayden, E.P., Singh, S.M., Sheikh, H.I., Kryski, K.R., Klein, D.N.Gene-environment correlations in the cross-generational transmission of parenting: Grandparenting moderates the effect of child 5-HTTLPR genotype on mothers' parenting. Soc Dev 2017, 26, 724-739.

24. Sturge-Apple, M. L., Davies, P. T., Martin, M. J., Cicchetti, D., Hentges, R. F.An examination of the impact of harsh parenting contexts on children's adaptation within an evolutionary framework. Dev. Psychol 2012, 48, 791-805.

25. Kagan, J., Snidman, N., Kahn, V., Towsley, S., Steinberg, L., Fox, N.A.The Preservation of Two Infant Temperaments into Adolescence. Monogr. Soc. Res. Child Dev. 2007, 72, 1-75.

26. Bakermans-Kranenburg, M. J., Van IJzendoorn, M. H. Handbook of Attachment Third Edition, Theory, Research, and Clinical Applications. 2016, Guilford, New York, NY.

27. Brigman, J.L., Mathur, P., Harvey-White, J., Izquierdo, A., Saksida, L.M., Bussey, T.J., Fox, S., Deneris, E., Murphy, D.L., Holmes, A.Pharmacological or Genetic Inactivation of the Serotonin Transporter Improves Reversal Learning in Mice. Cereb. Cortex, 2010, 20, 1955-1963.

28. Homberg, J.R., Lesch, P.K.Looking on the Bright Side of Serotonin Transporter Gene Variation. Biol. Psychiatry 2011, 69, 513-519. 
29. Jedema, H., Gianaros, P., Greer, P., Kerr, D.D., Liu, S., Higley, J.D., Suomi, S.J., Olsen, A.S., Porter, J.N., Lopresti, B.J., Hariri, A.R., Bradberry, C.W.Cognitive impact of genetic variation of the serotonin transporter in primates is associated with differences in brain morphology rather than serotonin neurotransmission. Mol. Psychiatry 2010, 15, 512-522.

30. Chronis-Tuscano, A., Raggi, V.L., Clarke, T.L., Rooney, M.E., Diaz, Y., Pian, J. Associations between Maternal Attention-Deficit/Hyperactivity Disorder Symptoms and Parenting. J. Abnorm. Child Psychol. 2008, 36.

31. Murray, C., Johnston, C.Parenting in mothers with and without attention-deficit/hyperactivity disorder. J. Abnorm. Psychol. 2006, 115, 52-61.

32. Deater-Deckard, K., Sewell, M.D., Petrill, S.A., Thompson, L.A.Maternal working memory and reactive negativity in parenting. Psychol. Sci. 2010, 21, 75-9.

33. Praschak-Rieder, N., Kennedy, J., Wilson, A.A., Hussey, D., Boovariwala, A., Willeit, M., Ginovart, N., Tharmalingam, S., Masellis, M., Houle, S., Meyer, J.H.Novel 5-HTTLPR allele associates with higher serotonin transporter binding in putamen: a [(11)C] DASB positron emission tomography study. Biol. Psychiatry 2007, 62, 327-31.

34. Johns, J.M., Joyner, P.W., McMurray, M.S., Elliott, D.L., Hofler, V.E., Middleton, C.L., Knupp, K., Greenhill, K.W., Lomas, L.M., Walker, C.H.The effects of dopaminergic/serotonergic reuptake inhibition on maternal behavior, maternal aggression, and oxytocin in the rat. Pharmacol. Biochem. Behav. $2005,81,769-785$.

35. Barofsky, A.L., Taylor, J., Tizabi, Y., Kumar, R., Jones-Quartey, K. Specific Neurotoxin Lesions of Median Raphe Serotonergic Neurons Disrupt Maternal Behavior in the Lactating Rat. Endocrinology 1983, 113, 1884-1893.

36. Galbally, M., Lewis, A.J., van Ijzendoorn, M., Permezel, M.The role of oxytocin in mother-infant relations: a systematic review of human studies. Harv. Rev. Psychiatry 2011, 19, 1-14.

37. Swain, J.E., Lorberbaum, J.P., Kose, S., Strathearn, L.Brain basis of early parent-infant interactions: psychology, physiology, and in vivo functional neuroimaging studies. J. Child Psychol Psychiatry 2007, $48,262-87$.

38. Skuse, D.H., Gallagher, L.Genetic influences on social cognition. Pediatr. Res.2011, 69, 85-91. 
39. Jørgensen, H., Riis, M., Knigge, U., Kjaer, A., Warberg, J.Serotonin receptors involved in vasopressin and oxytocin secretion. J. Neuroendocrinol. 2003, 15, 242-9.

40. Repetti, R.L., Taylor, S.E., Seeman, T.E. Risky families: family social environments and the mental and physical health of offspring. Psychol. Bull 2002, 128, 330-66.

41. Numan, M. Insel, T.R. Hormonal and Nonhormonal Basis of Maternal Behavior. The Neurobiology of Parental Behavior. 2003, Springer, New York, NY.

42. Keverne, E. B.Genomic imprinting, maternal care, and brain evolution. Horm. Behav. 2001, 40, 146155.

43. Hariri, A.R., Tessitore, A., Mattay, V.S., Fera, F., Weinberger, D.R.The amygdala response to emotional stimuli: a comparison of faces and scenes. Neuroimage 2002, 17, 317-23.

44. Canli, T., Lesch, K.P.Long story short: the serotonin transporter in emotion regulation and social cognition. Nat. Neurosci. 2007, 10, 1103-9.

45. Belsky, J., Beaver, K.M.Cumulative-genetic plasticity, parenting and adolescent self-regulation. J. Child Psychol. Psychiatry 2011, 52, 619-26.

46. Taylor, S. E., Way, B. M., Welch, W. T., Hilmert, C. J., Lehman, B. J., Eisenberger, N. IEarly family environment, current adversity, the serotonin transporter polymorphism, and depressive symptomatology. Biol. Psychiatry 2006, 60, 671- 676. 